\title{
Article \\ A 9-Bit 1-GS/s Hybrid-Domain Pseudo-Pipelined SAR ADC Based on Variable Gain VTC and Segmented TDC
}

\author{
Suping Bai ${ }^{1, *}$, Zhi Wan ${ }^{1} \mathbb{D}$, Peiyuan Wan ${ }^{2}$, Hongda Zhang ${ }^{2}$, Yongkuo Ma ${ }^{2}$, Xiaoyu Zhang ${ }^{2}$, Xu Liu ${ }^{2} \mathbb{0}$ \\ and Zhijie Chen ${ }^{2}$ \\ 1 The School of Electro-Optical Engineering, Changchun University of Science and Technology, \\ Changchun 130022, China; wan_zhi072139@163.com \\ 2 Faculty of Information Technology, College of Microelectronics, Beijing University of Technology, \\ Beijing 100124, China; wanpy@bjut.edu.cn (P.W.); feall3@emails.bjut.edu.cn (H.Z.); \\ mayk@emails.bjut.edu.cn (Y.M.); Zhangxiaoyu_emily@emails.bjut.edu.cn (X.Z.); \\ liuxu16@bjut.edu.cn (X.L.); chenzj@bjut.edu.cn (Z.C.) \\ * Correspondence: baisp@126.com; Tel.: +86-135-7887-2733
}

check for updates

Citation: Bai, S.; Wan, Z.; Wan, P.; Zhang, H.; Ma, Y.; Zhang, X.; Liu, X.; Chen, Z. A 9-Bit 1-GS/s

Hybrid-Domain Pseudo-Pipelined SAR ADC Based on Variable Gain VTC and Segmented TDC. Electronics 2021, 10, 2650. https://doi.org/ 10.3390 /electronics10212650

Academic Editors: Andrea Boni and Michele Caselli

Received: 26 September 2021

Accepted: 27 October 2021

Published: 29 October 2021

Publisher's Note: MDPI stays neutral with regard to jurisdictional claims in published maps and institutional affiliations.

Copyright: (c) 2021 by the authors. Licensee MDPI, Basel, Switzerland. This article is an open access article distributed under the terms and conditions of the Creative Commons Attribution (CC BY) license (https:/ / creativecommons.org/licenses/by/ $4.0 /)$.

\begin{abstract}
This paper presents a 9-bit $1 \mathrm{GS} / \mathrm{s}$ successive approximation register (SAR) analog-todigital converter (ADC). In this hybrid architecture, the pseudo-pipeline operation is realized, which increases the sampling rate effectively. The ADC adopts two key technologies: the variable gain voltage-to-time converter (VTC), which ensures the linearity is not sacrificed; the segmented time-todigital converter (STDC), which further improves the linearity of time domain quantization. The prototype ADC is simulated in a standard 65-nm CMOS process with an active area of $0.038 \mathrm{~mm}^{2}$. The simulated SNDR and SFDR are 44.3 and $58 \mathrm{~dB}$ with a sampling rate of $1 \mathrm{GS} / \mathrm{s}$. The FoMW and FoMS are $24.7 \mathrm{fJ} /$ conv-step and $150.7 \mathrm{~dB}$, respectively.
\end{abstract}

Keywords: analog-to-digital converter (ADC); hybrid-domain quantization; high speed; voltage-totime converter (VTC); time-to-digital converter (TDC)

\section{Introduction}

The higher data receiving rate in communication systems increases the demand for high-speed ADCs in recent years. SAR ADCs are popular because of the excellent power efficiency. To increase the sampling rate of SAR ADCs, time interleaving technique [1-4] is used by multiplexing several converters in parallel. As the fastest architecture, pipelined ADC is combined to further contribute to the conversion speed of time interleaving ADC [5]. However, the time interleaving multiplies power consumption and the area, the mismatch between channels magnifies dynamic errors as well. Compared with traditional SAR ADCs, time-domain quantization has better performance in terms of high-speed conversion, lowvoltage design, and reduction of mismatch. In terms of high-speed conversion, the SAR ADC needs to perform charge redistribution every time it completes 1 bit of quantization. In order to ensure that the voltage is fully established, it is generally necessary to reserve a sufficient conversion time. Especially when the number of quantization bits is high, the capacitance in the Digital-to-Analog Converter (DAC) will also increase exponentially, which limits the improvement of the conversion speed. In the time domain quantization, according to the working principle of TDC, each conversion cycle can complete multi-bit quantization. Compared with the single-bit quantization method based on the comparator, the number of charge redistribution is reduced, and the sampling rate is increased, accordingly. Analog-to-digital converters (ADCs) employing time-to-digital converters (TDCs) combined with traditional voltage-domain ADCs have recently been reported [6] to show reasonable power efficiency. Time-domain quantization [7-9] is suitable for the high-speed SAR ADCs design, because the delay cell is faster due to the process scaling, but the operation in voltage-domain and time-domain is performed in sequence, with the 
improvement in speed limited. There are three critical timings in a typical SAR ADCs: the comparator decision/resolving time, the CDAC settling time, and the digital logic delay $[10,11]$. The comparator decision/resolving time is a function of the input voltage, but the relationship is not utilized in most ADCs.

In this work, a two-stage SAR ADC is achieved by utilizing the voltage-domain and time-domain conversion and pseudo-pipelined operation. Different from the conventional hybrid quantization method, the signal is converted in voltage- and time-domain almost simultaneously, which increases the sampling rate obviously. In Section 2 the architecture and timing are introduced. Moreover, a variable gain voltage-to-time converter (VTC) and a segmented time-to-digital converter (STDC) are proposed in Section 3 to overcome the nonlinearity of the time-domain conversion. In Section 4 the simulation results are presented. Finally, the conclusion is given in Section 5.

\section{Proposed ADC Architecture}

\subsection{Architecture}

The proposed high-speed ADC architecture, as shown in Figure 1, comprises a voltage ADC (VADC), a time ADC (TADC), and a digital logic. The converter has a total of 9bit output. The 2-MSB quantization is completed in the voltage-domain and the 7-LSB quantization is completed in the time domain. It is expected to achieve a sampling rate of $1 \mathrm{GS} / \mathrm{s}$.

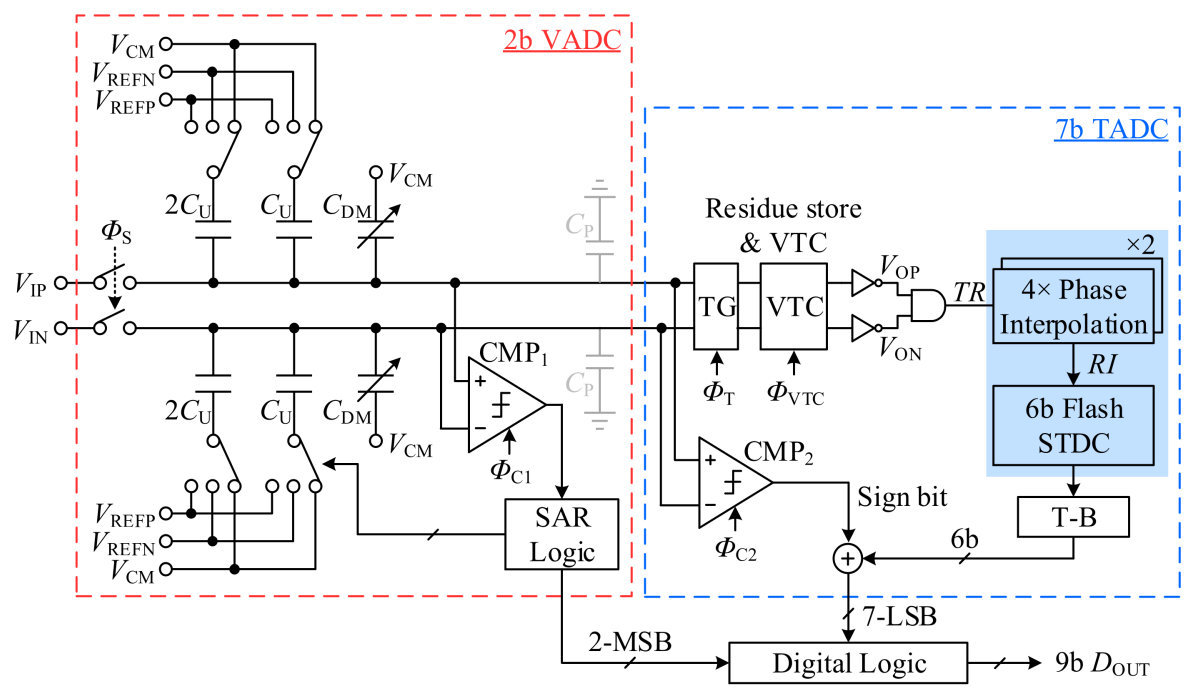

Figure 1. Architecture of the proposed two-stage ADC.

The voltage-domain component operation as a traditional 2-bit SAR ADC is used to quantify MSBs. The input signal uses a differential form to reduce common-mode noise and the even harmonics of the output and utilizes the capacitor's top plate to sample the input to increase the $\mathrm{ADC}$ conversion speed. In the time-domain component, the voltage residue is converted to a time-domain signal through the VTC, and the STDC is used to complete the LSBs quantization. The input of the comparator $\mathrm{CMP}_{2}$ is connected to the upper plate of the capacitor in the VADC to compare the magnitude of the voltage residue at both ends of the differential. The output of the VADC is connected to the VTC through a transmission gate; an AND gate and an inverter are added to the next stage. The conversion results of the two time-domain signals are combined into one as the input of the STDC. After the voltage residue is converted into the corresponding time signal TR, a 7-LSB quantization result is produced under the action of the STDC. The thermometer-to-binary converter circuit (T-B) is used to convert the output of the STDC into binary and superimpose it with the sign bit, which produces the conversion result of the TADC. 


\subsection{Timing}

The timing diagram of the proposed ADC is illustrated in Figure 2. During the sampling phase $\Phi_{\mathrm{S}}$, the input voltages are sampled into the DAC in VADC. When $\Phi_{\mathrm{C} 1}$ is high level, the sampling completed and the comparator $\mathrm{CMP}_{1}$ converts the MSBs. The comparator operates twice, and the quantization of the voltage-domain is completed. Before the next convert cycle, the voltage residue is generated by the CDAC and transferred to the TADC while $\Phi_{\mathrm{T}}$ is low; the transmission gate in the VTC is turned off. Meanwhile, $\Phi_{\mathrm{C} 2}$ goes high; the comparator $\mathrm{CMP}_{2}$ discriminates the polarity of the voltage residue and generates a sign bit; the conversion result $T R$ is outputted by VTC under the control of $\Phi_{\mathrm{VTC}}$. The rising edge interval of $\Phi_{\mathrm{VTC}}$ and TR is the inputs of the STDC. Utilizing a phase interpolation circuit, the 4-bit time signal $R I$ [3:0] is extended by $T R$ and the interval between adjacent RI signals is equal to one-fourth of the unit delay td of TDC. Consequently, the TADC completes the 7-bit quantization expeditiously.

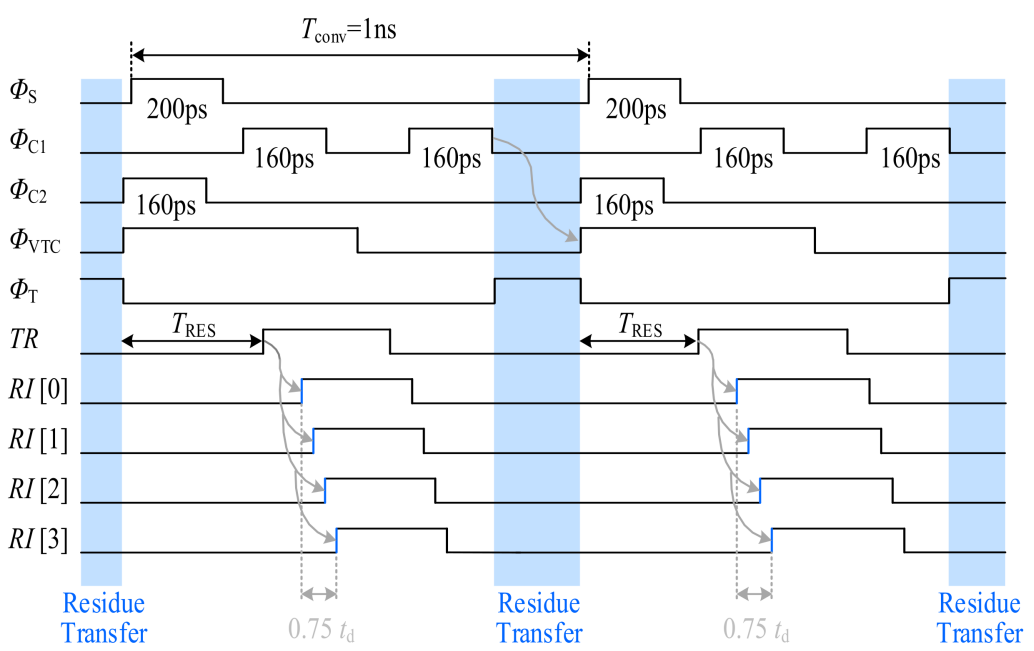

Figure 2. Timing diagram of the proposed two-stage ADC.

Since the negative edge of the clock $\Phi_{\mathrm{T}}$ is slightly earlier than the positive edge of the sampling clock $\Phi_{\mathrm{S}}$, the connection between VTC and VADC was disconnected before the next sampling cycle. The last conversion residue is not affected by the new input voltage. Therefore, the TADC converts the previous residue in time-domain, and the VADC samples the next voltage input signal and determines the MSBs.

The pseudo-pipeline operation is realized. The voltage-domain and the time-domain quantization are carried out almost at the same time, and the two-stage conversion process is completed in only one clock cycle, which improves the sampling rate of the ADC effectively.

\section{Circuit Implementation}

\subsection{Capacitive DAC}

A 2-bit capacitive DAC samples input voltage and generates voltage residue. The circuit architecture of the capacitive DAC is shown in Figure 3. The load of the DAC in the conventional SAR ADC is a comparator. However, the DAC in the VADC is connected to the VTC and the comparator $\mathrm{CMP}_{2}$; an additional parasitic capacitor is generated between the top plate and the ground. The parasitic capacitor is equivalent to increasing the dummy capacitance $C_{\mathrm{DM}}$. According to the principle of charge redistribution, if there is an error in the dummy capacitor, the voltage of the top plate will change incorrectly, and the voltage residue is affected. To reduce the error, this paper divides the $C_{\mathrm{DM}}$ into two parts: one is a capacitor of $3 C$, and its bottom plate is always connected to the common mode voltage; the other contains 4 MOS capacitors with digital adjustment, the capacitance value ratio is 
1:2:4:8. The capacitance of the $C_{\mathrm{DM}}$ can be adjusted by changing the 4-bit control signal $\mathrm{D}_{\mathrm{CAL}}[3: 0]$ to ensure that the $C_{\mathrm{DM}}$ is near $4 \mathrm{C}$.

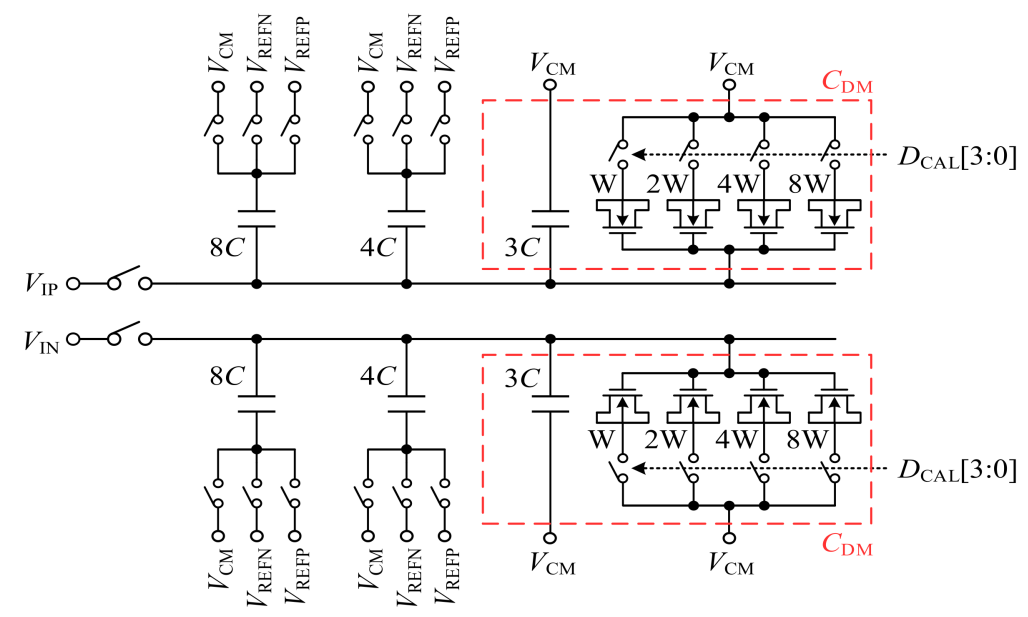

Figure 3. Capacitive DAC.

\subsection{Voltage-to-Time Converter (VTC)}

The VTC builds the interface between the voltage-domain and time-domain conversions. The circuit structure of the traditional voltage type VTC is shown in Figure 4a. The input voltage is directly connected to the output node through the switch. In the sampling phase, the voltage input $V_{\mathrm{IP}}$ and $V_{\mathrm{IN}}$ are collected into the parasitic capacitance $C_{\mathrm{P}}$. During the conversion phase, the top switch is turned off, and $\mathrm{M}_{1}-\mathrm{M}_{4}$ are turned on. Since the gate voltages of $\mathrm{M}_{1}$ and $\mathrm{M}_{2}$ are provided by the DC bias $V_{\mathrm{b}}$, both ends will discharge $C_{\mathrm{P}}$ with the same current $I_{C}$. As shown in Figure $4 \mathrm{~b}$, different input voltages change the initial point of the discharge. After the voltage drops to $V_{\mathrm{IL}}$, TDC is triggered and the conversion from voltage to time is completed. The proposed structure is based on the voltage type VTC, and the operation principle is shown in Figure $5 \mathrm{a}, \mathrm{b}$. When $\Phi_{\mathrm{T}}$ is low, VTC collects the voltage residue, the output $V_{\mathrm{P}}$ and $V_{\mathrm{N}}$ of the VADC are transmitted to the nodes $V_{\mathrm{RP}}$ and $V_{\mathrm{RN}}$ through the source followers to pre-charge the dummy capacitor. When $\Phi_{\mathrm{T}}$ is high, the VTC converts the voltage residue and CMOS switch $\mathrm{M}_{5}-\mathrm{M}_{8}$ are turned off. Voltage $V_{\mathrm{RP}}$ and $V_{\mathrm{RN}}$ decrease at a constant rate of discharge current provided by $\mathrm{M}_{9}$ and $\mathrm{M}_{10}$. The relationship between time and output voltage $V_{\mathrm{OP}}$ can be expressed as:

$$
V_{\mathrm{OP}}=V D D-\frac{K\left(V_{\mathrm{CAL}}-V_{\mathrm{TH}}\right)^{2}}{C_{\mathrm{P}}} \cdot\left(1+\lambda V_{\mathrm{OP}}\right) t
$$

Here $K=\frac{1}{2} \mu_{\mathrm{n}} C_{\mathrm{ox}} W / L$, assuming $K_{\mathrm{VTC}}=\frac{K\left(V_{\mathrm{CAL}}-V_{\mathrm{TH}}\right)^{2}}{C_{\mathrm{P}}}$, then $V_{\mathrm{OP}}=V D D-K_{\mathrm{VTC}}$ $\left(1+\lambda V_{\mathrm{OP}}\right) t$, the relationship between the output time and the input voltage is expressed as:

$$
T_{\text {out }}=\frac{V D D-V_{\mathrm{IL}}}{K_{\mathrm{VTC}}\left(1+\lambda V_{\mathrm{IL}}\right)}+\frac{1}{\lambda K_{\mathrm{VTC}}}-\frac{\lambda V D D+1}{\lambda^{2} K_{\mathrm{VTC}}\left(V_{P 0}+\frac{1}{\lambda}\right)}
$$

where $V_{\mathrm{IL}}$ is the triggered voltage of the inverter, and $V_{\mathrm{P} 0}$ is the input voltage. A wider output range of VTC is desired to reduce the requirement for TDC. $\mathrm{M}_{11}$ and $\mathrm{M}_{\mathrm{CAL}}$ are utilized as voltage dividers to reduce the gate voltage $V_{\mathrm{CAL}}$ of $\mathrm{M}_{9}$ and $\mathrm{M}_{10}$, thereby reduce the discharge current and extend the output time range. $\mathrm{M}_{\mathrm{CAL}}$ contains three parallel transistors, by switching the 3-b codes Cal [2:0], $V_{\mathrm{CAL}}$ and charging current are changed. The linearity of VTC will be severely affected by PVT, e.g., $V_{\mathrm{TH}}, \mu_{n}$. It can be seen from the above formula that $V_{\mathrm{TH}}, \mu_{\mathrm{n}}$ are included in $K_{\mathrm{VTC}}$, so the influence of PVT on the conversion characteristics of VTC can be eliminated by changing $V_{\mathrm{CAL}}$. 


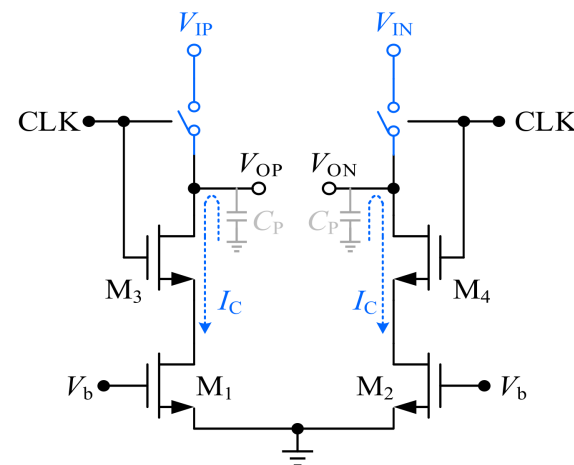

(a)

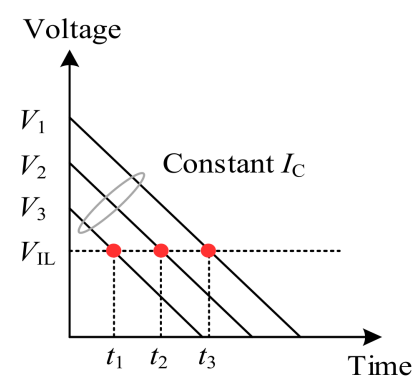

(b)

Figure 4. (a) VTC circuit based on variable voltage. (b)operation principle.

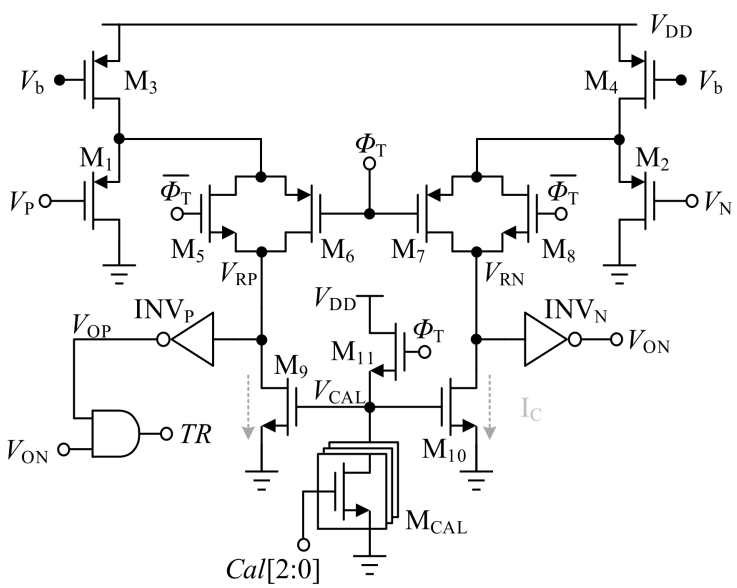

(a)

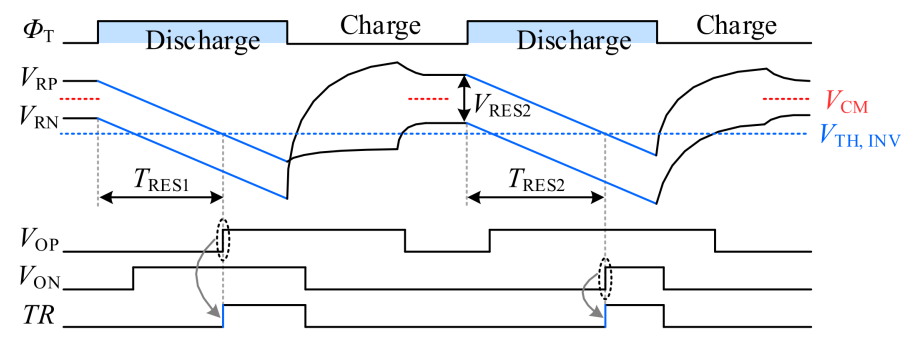

(b)

Figure 5. (a) Design of the proposed VTC and (b) timing diagram.

\subsection{6-Bit TDC}

Due to the secondary effect of the circuit, the output of VTC is nonlinear. As shown in Figure $6 b$, the slope of the conversion characteristic curve decreases as the voltage rises. In order to reduce the impact of VTC nonlinearity, the conversion from the time-domain to the digital code based on the segment delay line TDC is proposed. The main principle is to split the delay line into two parts, each part has a different delay and resolution to create a nonlinear TDC artificially. If the nonlinearity of VTC and TDC can compensate each other, the linearity of the digital output will be improved. The shaded area between the VTC and TDC characteristic curves means the nonlinear error. 


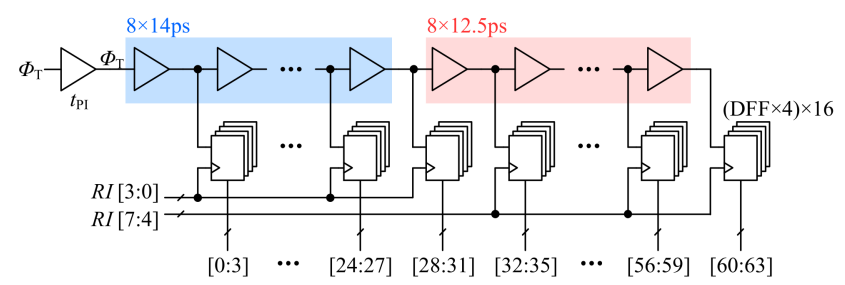

(a)

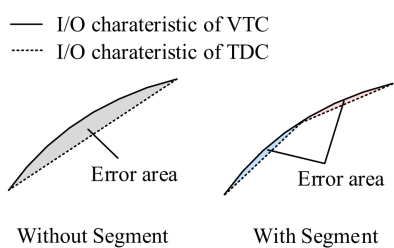

(b)

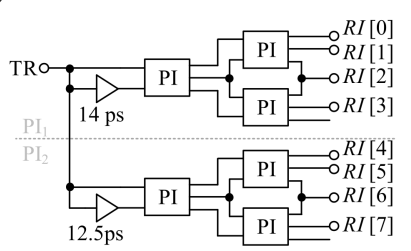

(c)

Figure 6. (a) Segment TDC. (b) Conversion characteristic of the VTC. (c) circuit of the phase interpolation.

With the segmented TDC, the total error area is significantly reduced. The delay line circuit of the segmented TDC is shown in Figure 6a. The delay line is divided into two parts; each part contains 8 delay units, and the delays are 14 ps and 12.5 ps, respectively. The overall delay is only 212 ps. It adopts a 4 times phase interpolation structure, the output of each delay line is connected with the input terminals of $4 \mathrm{D}$-flip-flops, and the clock terminal of D flip-flop is connected with the output of the phase interpolation circuit. Because of the differences in the delays, two-phase interpolation circuits are needed to generate different time intervals, as shown in Figure 6c. The lower 4 bits of RI are generated by the first phase interpolation circuit $\mathrm{PI}_{1}$ (the maximum time difference is $14 \mathrm{ps}$ ), which is connected to the clock terminal of the first half of the TDC; the upper 4 bits of RI are generated by the second phase interpolation circuit in the second half of the TDC. Using this phase interpolation technology, the resolution and conversion speed of the TDC can be improved, and the linearity of time-domain quantization is optimized.

\section{Experimental Results}

Figure 7 show the ADC layout in a standard 1P9M 65-nm TSMC with an active area of $0.038 \mathrm{~mm}^{2}$. The simulated total power consumption of the ADC is $3.277 \mathrm{~mW}$ with a $1-\mathrm{V}$ supply. The power consumption breakdown is shown in Figure 8. It can be broken down as follows: the bootstraps use $9 \%$ of the power; the VADC and the VTC uses 36\%; the digital circuits use $4 \%$; and the TDC use $51 \%$.

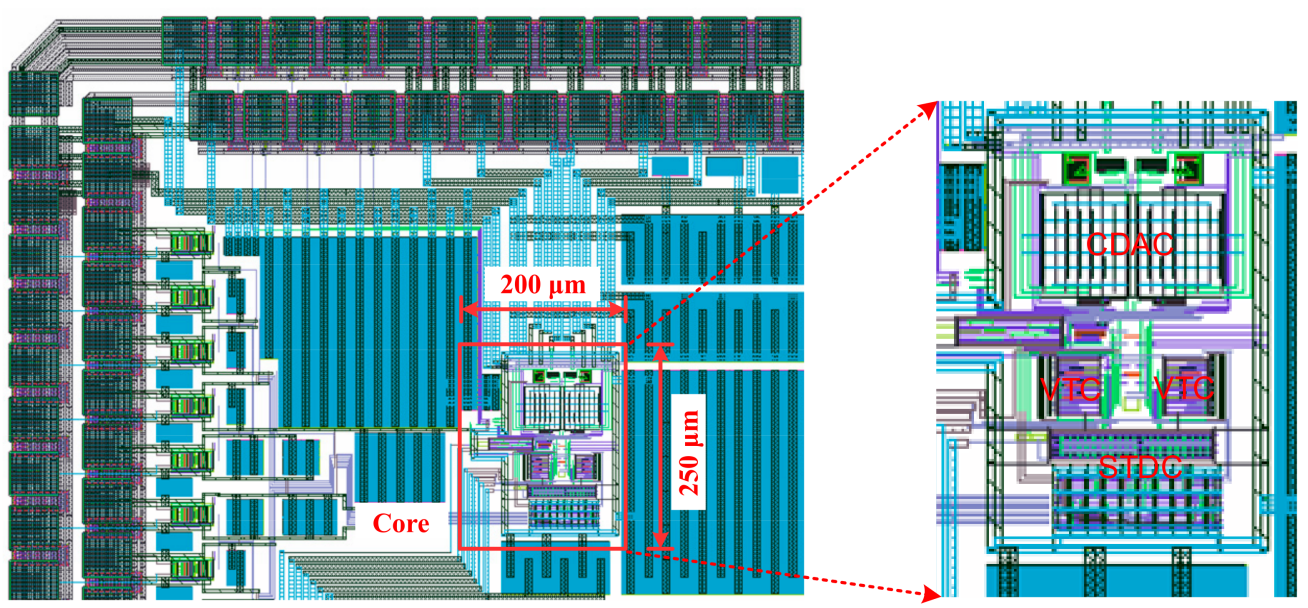

Figure 7. Layout of the proposed ADC. 


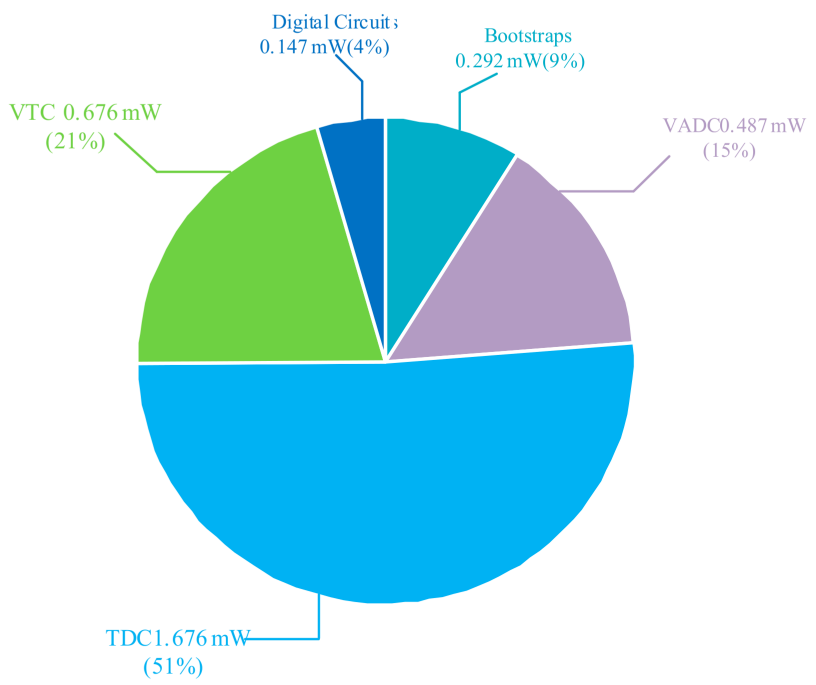

Figure 8. Pie chart of the ADC power distribution.

Figure 9 illustrates the simulated spectrum with a conversion rate of 1-GS/s and an input frequency of $163 \mathrm{MHz}$. The simulated signal-to-noise-and-distortion ratio (SNDR) is $44.3 \mathrm{~dB}$. The static performance of the DNL and INL by post-layout are $+1.2 /-1.0$ and $+2.79 /-2.89$ LSB, respectively, as shown in Figure 10. The error is mainly caused by the nonlinearity of the TADC. The parasitic capacitance of the post-layout affects the conversion gain of the VTC and the unit delay of the TDC, resulting in time-domain quantization errors and missing codes in TDC errors. Although the segmented TDC can compensate for the conversion characteristics of the VTC to a certain extent, it is still unable to eliminate its nonlinear error.

When the sampling rate input $\mathrm{F}_{\mathrm{IN}}$ is changed from $90 \mathrm{MHz}$ to $390 \mathrm{MHz}$, the postsimulated SNDR is maintained above $43.8 \mathrm{~dB}$, as shown in Figure 11. The ADC attains a FoM of $24.3 \mathrm{fJ} /$ conv-step at Nyquist rate. Table 1 summarizes the ADC performance and shows the comparison with the recently published GS/s ADCs.

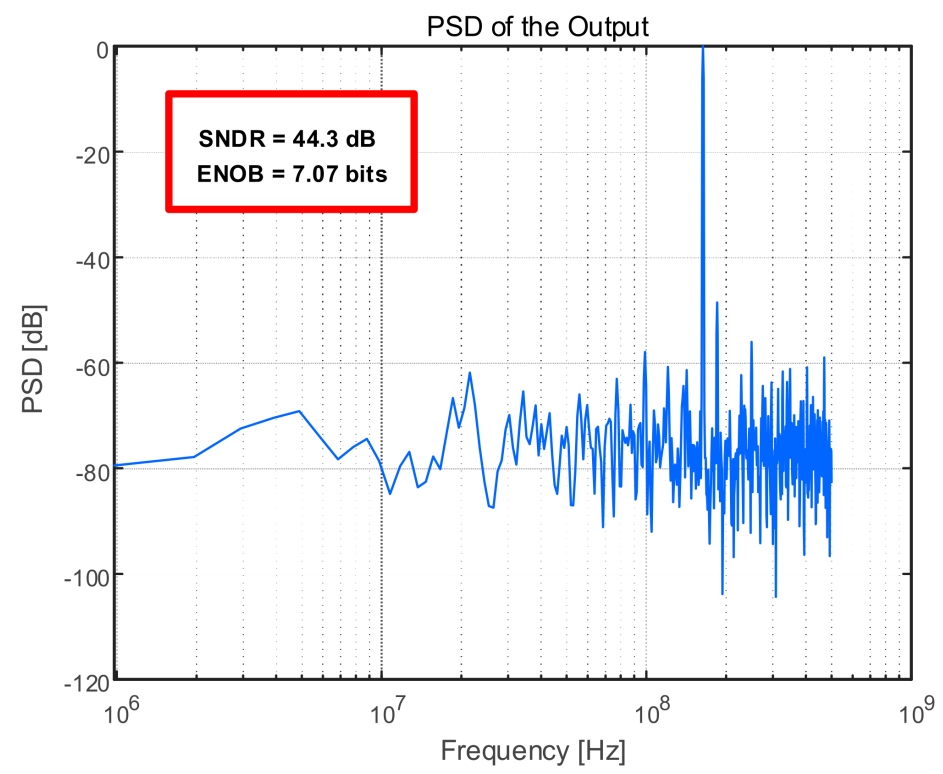

Figure 9. Power spectral density of the proposed ADC. 

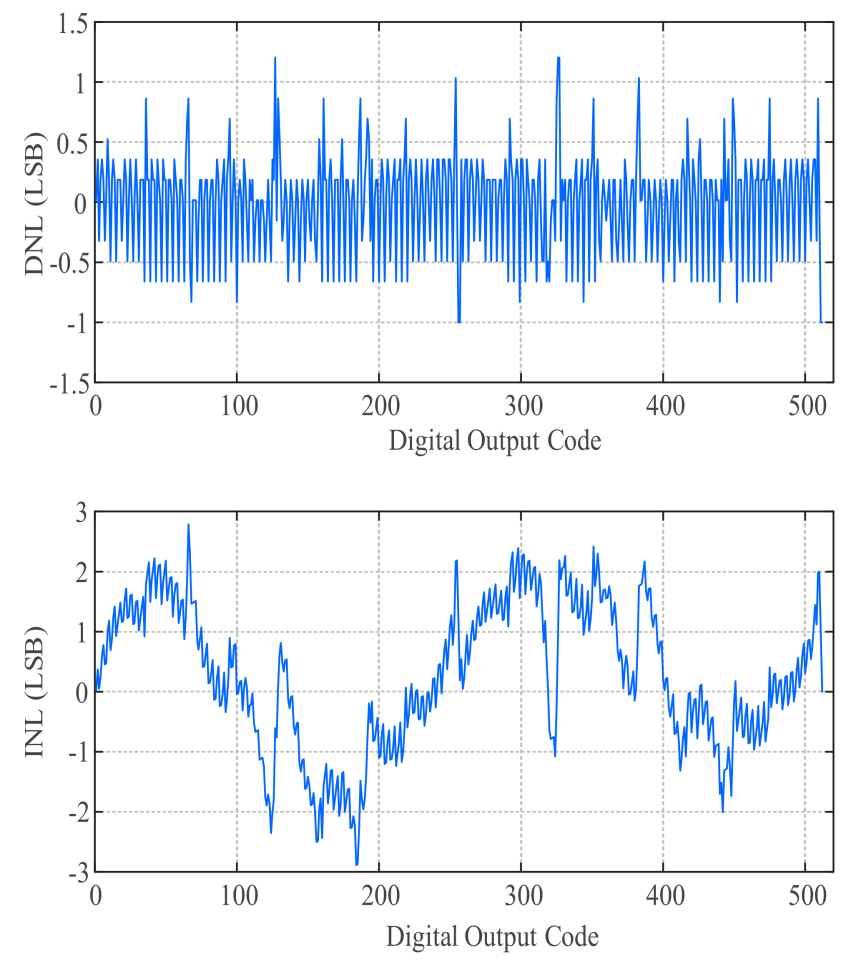

Figure 10. Post-simulated DNL and INL.

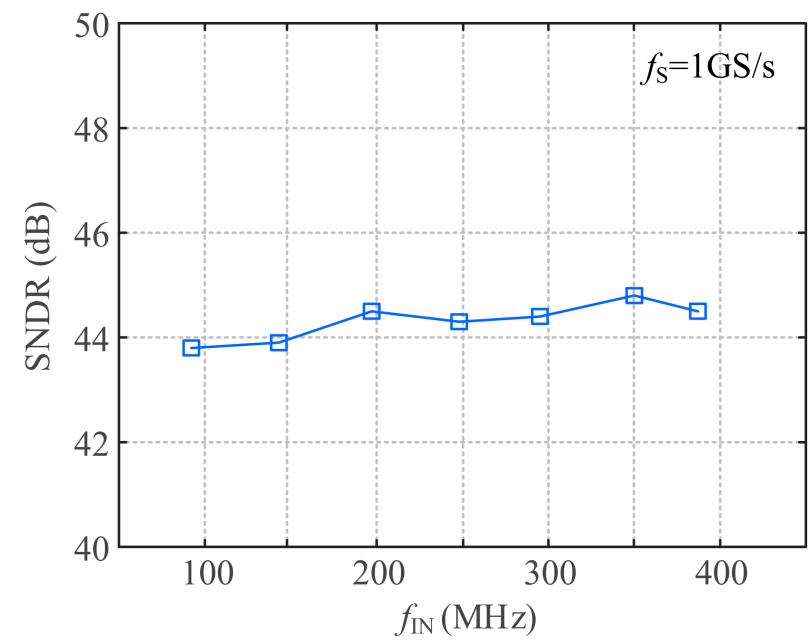

Figure 11. Post-simulated SNDR versus input frequency.

Table 1. Performance Summary.

\begin{tabular}{ccccc}
\hline & $\begin{array}{c}\text { ISSCC17 [2] } \\
\text { Measured }\end{array}$ & $\begin{array}{c}\text { A-SSCC18 [3] } \\
\text { Measured }\end{array}$ & $\begin{array}{c}\text { ISCAS18 [5] } \\
\text { Simulated }\end{array}$ & $\begin{array}{c}\text { This Work } \\
\text { Simulated }\end{array}$ \\
\hline Type & TI-SAR & TI-pip-SAR & Pip-SAR & SAR-TDC \\
CMOS (nm) & 65 & 65 & 28 & 65 \\
Active Area (mm ${ }^{2}$ ) & 1.7 & 0.128 & 0.175 & 0.038 \\
Supply Voltage (V) & 1.2 & $1.2 / 2.5$ & 1 & 1 \\
Power (mW) & 44.6 & 22 & 16.02 & 3.277 \\
Sampling Rate (GS/s) & 2.8 & 1 & 0.8 & 1 \\
SNDR (dB) & 48.2 & 54.3 & 60.9 & 44.3 \\
FoMwalden[fj/c-s] & 75.8 & 43.7 & 22.5 & 24.3 \\
\hline
\end{tabular}




\section{Conclusions}

This paper presents a 9-bit 1-GS/s, single-channel, hybrid, voltage-time, pseudopipelined ADC in a 65-nm CMOS technology. The new hybrid ADC combines the high accuracy of the voltage-domain quantification and the high speed of the time-domain quantification to satisfy the need for high-speed ADCs, reducing the accuracy requirements of the comparator, converting the voltage signal into a time signal, and performing lowbit quantization in the time domain. A variable gain VTC, and segmented delay line TDC based on phase interpolation technique were proposed. The matching and linear conversion between VTC and TDC are guaranteed, and the quantization of the 6-LSB of the time domain only uses 212 ps resulting in an efficient operation. Using a 1-V supply, the prototype ADC dissipates a total power of $3.277 \mathrm{~mW}$ and achieves the Nyquist ENOB of 7.07 bits. The Nyquist FOM is $24.3 \mathrm{fJ} /$ conversion-step. This paper shows that the proposed ADC is a good candidate for the implementation of high-speed and high-resolution ADCs.

Author Contributions: Conceptualization, Z.W., S.B.; Data curation, H.Z. and Y.M.; Investigation, P.W., X.Z. and X.L.; Methodology, H.Z., Z.W.; Y.M. and X.Z.; Resources, P.W. and Z.C.; Writingoriginal draft, Z.W., H.Z. and Y.M.; and Writing-review and editing, Z.W., H.Z., P.W. and Z.C. All authors have read and agreed to the published version of the manuscript.

Funding: This work was supported by Beijing Natural Science Foundation Project No. 4202010, and National Natural Science Foundation of China, Key Project No.61731019.

Data Availability Statement: The data presented in this study are available on request from the corresponding author.

Conflicts of Interest: The authors declare no conflict of interest.

\section{References}

1. Kull, L.; Luu, D.; Menolfi, C.; Braendli, M.; Francese, P.A.; Morf, T.; Kossel, M.; Cevrero, A.; Ozkaya, I.; Toifl, T. A 24-to-72GS/s 8b time-interleaved SAR ADC with 2.0-to-3.3pJ/conversion and $>30 \mathrm{~dB}$ SNDR at nyquist in 14nm CMOS FinFET. In Proceedings of the 2018 IEEE Symposium on VLSI Circuits, Honolulu, HI, USA, 18-22 June 2018; pp. 358-360. [CrossRef]

2. Keane, J.P.; Guilar, N.J.; Stepanovic, D.; Wuppermann, B.; Wu, C.; Tsang, C.W.; Neff, R.; Nishimura, K. 16.5 An 8 GS/s timeinterleaved SAR ADC with unresolved decision detection achieving $-58 \mathrm{dBFS}$ noise and $4 \mathrm{GHz}$ bandwidth in $28 \mathrm{~nm}$ CMOS. In Proceedings of the 2017 IEEE International Solid-State Circuits Conference (ISSCC), San Francisco, CA, USA, 5-9 February 2017; pp. 284-285. [CrossRef]

3. Zhu, Y.; Chan, C.-H.; Martins, R. An 11b 1GS/s Time-Interleaved ADC with Linearity Enhanced T/H. In Proceedings of the 2018 IEEE Asian Solid-State Circuits Conference (A-SSCC), Tainan, Taiwan, 5-7 November 2018; pp. 1-2. [CrossRef]

4. Chen, Y.; Wang, J.; Hu, H.; Ye, F.; Ren, J. A Time-Interleaved SAR Assisted Pipeline ADC With a Bias-Enhanced Ring Amplifier. IEEE Trans. Circuits Syst. II Express Briefs 2017, 65, 1584-1588. [CrossRef]

5. Chen, Y.; Ni, Z.; Cao, Y.; Ye, F.; Ren, J. A 800 MS/s, 12-Bit, Ringamp-Based SAR assisted Pipeline ADC with Gain Error Cancellation. In Proceedings of the 2018 IEEE International Symposium on Circuits and Systems (ISCAS), Florence, Italy, 27-30 May 2018; pp. 1-4. [CrossRef]

6. Muhlestein, J.; Leuenberger, S.; Sun, H.; Xu, Y.; Moon, U.-K. A 73dB SNDR 20MS/s 1.28mW SAR-TDC using hybrid two-step quantization. In Proceedings of the 2017 IEEE Custom Integrated Circuits Conference (CICC), Austin, TX, USA, 30 April-3 May 2017; pp. 1-4. [CrossRef]

7. Su, Z.; Wang, H.; Zhao, H.; Chen, Z.; Wang, Y.; Dai, F.F. A 280MS/s 12b SAR-Assisted Hybrid ADC with Time Domain Sub-Range Quantizer in 45nm CMOS. In Proceedings of the 2019 IEEE Custom Integrated Circuits Conference (CICC), Austin, TX, USA, 14-17 April 2019; pp. 1-4. [CrossRef]

8. Zhang, M.; Chan, C.-H.; Zhu, Y.; Martins, R.P. 3.5 A 0.6V 13b 20MS/s Two-Step TDC-Assisted SAR ADC with PVT Tracking and Speed-Enhanced Techniques. IEEE 2019, 66-68. [CrossRef]

9. Liu, C.-C. 27.4 A 0.35mW 12b 100MS/s SAR-assisted digital slope ADC in 28nm CMOS. In Proceedings of the 2016 IEEE International Solid-State Circuits Conference (ISSCC), San Francisco, CA, USA, 31 January-4 February 2016; pp. $462-463$. [CrossRef]

10. Ramkaj, A.T.; Strackx, M.; Steyaert, M.S.J.; Tavernier, F. A 1.25-GS/s 7-b SAR ADC With 36.4-dB SNDR at 5 GHz Using Switch-Bootstrapping, USPC DAC and Triple-Tail Comparator in 28-nm CMOS. IEEE J. Solid-State Circuits 2018, 53, 1889-1901. [CrossRef]

11. Lyu, Y. A 4-GS/s 39.9-dB SNDR 11.7-mW Hybrid Voltage-Time Two-Step ADC With Feedforward Ring Oscilla-tor-Based TDCs. IEEE JSSC 2020, 55, 1807-1818. 